\title{
The challenges in producing high-quality clinical evidence
}

\author{
W. Scott Beattie, MD, PhD
}

Received: 29 October 2015/Accepted: 12 November 2015/Published online: 15 December 2015

(C) Canadian Anesthesiologists' Society 2015

"Tis but thy name that is my enemy......"
Romeo and Juliet, Act II Scene II

In 1996, David Sackett opined that "Evidence-based medicine $[\mathrm{EBM}]$ means integrating individual clinical expertise with the best available external clinical evidence from systematic research". Clinicians, educators, investigators, and administrators seeking to improve clinical care have adopted these tenets, and now, EBM fuels activities that aim to produce high-quality clinical evidence.

The adoption of EBM has resulted in an ever expanding number of best practice guidelines, ${ }^{1}$ pay for performance rules, and/or public policies. ${ }^{2}$ Sadly for perioperative medicine, this process of research, knowledge translation, and the resultant changes to the perioperative evidence base has not yielded the types of clinical improvements that many would have predicted. ${ }^{3}$ There are various explanations for these failures, but in my opinion, the blame lies mostly in our research methodology. ${ }^{4}$ Improving the quality of clinical investigation requires more than just a process of randomization. ${ }^{5} \mathrm{We}$ are challenged to conduct efficient, expedient, and adequately powered trials, pursuing biologically sound hypotheses that enhance clinically important, clearly defined, outcomes.

While the ideal of EBM is highly promoted, in practice, there are numerous obstacles. The biggest problem is classic economics; demand exceeds supply. The notion that each clinical question, of which there are too many to count, can be answered by a meta-analysis and/or systematic reviews of multiple randomized controlled trials (RCTs) is naive. In fact, there are only a few

W. S. Beattie, MD, PhD ( $₫)$

Department of Anesthesia, University Health Network, University of Toronto, Toronto, ON, Canada

e-mail: scott.beattie@uhn.ca examples of studies in perioperative medicine that lead to the highest quality recommendations. ${ }^{6,7}$ Our failures are examples where clinical recommendations were based on information that was prone to any number of biases. ${ }^{8}$ The challenge now is to produce quality evidence.

The RCT has become synonymous with unbiased evidence. Nevertheless, the ability to generate and reproduce a high-quality RCT is constrained by a second economic reality, funding. Funding is a constant impediment that limits perioperative clinical research and thus hinders EBM. The need for multidisciplinary personnel, infrastructure, increasing layers of bureaucracy, privacy issues, and the time to complete studies has added to the costs of conducting any study. At the same time, the amount of peer-reviewed funding available to Canadian investigators is stagnant or decreasing. ${ }^{9}$ Another aspect of the escalating expense for research is the realization that, to pursue clinically important outcomes, interventional trials require study samples large enough to capture meaningful and reproducible differences; often a trial will require several thousand patients. ${ }^{10}$ In Transforming Clinical Research in the United States: Challenges and Opportunities, it has been estimated that global interventional trials can cost more than 300 million dollars. ${ }^{11}$ In truth, this number overestimates the costs of recent perioperative trials by over 20 fold, but costs will continue to increase and trial opportunities must be optimized.

This special edition of the Journal acknowledges an important step in this direction, a discussion to define clinically important and patient-centred outcomes. ${ }^{12,13}$ Meta-analysis of well-conducted RCTs is promoted as the pinnacle of evidence, in other words, findings that are reproducible. ${ }^{14}$ Future meta-analyses will be stronger when every trial pursues a set of universally accepted outcomes. 
Why is there concern? Most clinicians assume that outcomes are black and white-i.e., we know what they mean and which ones are important. Surprisingly, the objective definitions of many clinical outcomes are ambiguous, and ambiguity can lead to misinterpretation. Let us consider the definition of death, which would seem to us to be the easiest of outcomes to define. Nevertheless, is it important to collect in-hospital deaths or deaths at 30 days after the intervention? In cardiovascular trials, for example, is it important to distinguish between cardiac and non-cardiac death? If yes, how would you define cardiac death? Would you define it as the absence of other causes that may have occurred silently, or is it necessary to use objective (perhaps biomarker) evidence? Presently, these types of questions remain unanswered and lack any kind of consensus. It is therefore not surprising to find discordant results when the definitions for both baseline characteristics and postoperative outcomes of two prominent quality improvement projects (i.e., National Surgical Quality Improvement Program and the Vascular Quality Initiative) were applied to a common database. We need look no further to illustrate the pressing need to define most accepted, clinically important outcomes. ${ }^{15}$ Even though we know the name (i.e., of the outcome), clearly, how it is defined is more important.

Universally, the most important clinical and patientcentred outcomes are often the most infrequent outcomes. This reality can make conducting a trial infeasible simply because of the required sample size. In most modern trials, investigators therefore utilize both surrogate and/or composite outcomes to reduce the size of the study cohort. While these instruments are both common and necessary, investigators have encountered problems when using them to interpret the results of numerous trials.

As an example, the Acute Kidney Injury Network (AKIN) or Risk Injury FaiLurE (RIFLE) criteria for acute renal failure are high profile examples of surrogate outcomes that use objective changes in biomarkers (i.e., creatinine and urine output) to define outcomes. ${ }^{16}$ Both AKIN and RIFLE have gained acceptance since prospective observational studies have shown significant associations with important, but infrequent, outcomes such as dialysis and death. The magnitude of these associations is illustrated in a meta-analysis to assess the variability and precision of the AKIN criteria. In this study of eight trials and more than 50,000 patients, mild acute kidney injury, as defined by an increase in creatinine of $>25 \mathrm{mmol} \cdot \mathrm{L}^{-1}$ (i.e., the surrogate), was seen in $17 \%$ of patients. ${ }^{17}$ This degree of elevation in creatinine was associated with a significant two-fold increase in near-term death. Nevertheless, the death rate seen in this analysis was $1.2 \%$ (619 patients), an incidence that was 14-fold smaller than AKIN-defined renal failure. Since the event rates of the surrogate are more frequent, a smaller sample size can be recruited, but we need to remain cognizant of the fact that over $95 \%$ of patients who experienced the surrogate outcome did not experience the hard outcome.

Smaller cohorts can also be recruited when a composite outcome is used as the primary outcome. Nevertheless, when composites are utilized, they require close scrutiny and cautious interpretation. The name (i.e., of the composite) may be the same, but how it is defined is often inconsistent and prone to manipulation, which can lead to confusing results. ${ }^{18}$ Composite outcomes are often constructed post hoc and may include factors with a diverse range of clinical importance, which has led to conflicting or biased results. ${ }^{19,20}$

A prime example of such an outcome is major adverse cardiac events (MACE). ${ }^{21}$ The MACE outcome is popular and widely utilized, but its components vary enormously between studies. A systematic review comparing the efficacy of drug-eluting stents (DES) and bare-metal stents $(\mathrm{BMS})^{22}$ found ten different components used to define MACE. There were as few as three and as many as seven components used to define MACE. The factors ranged in clinical importance from the clinical need to revascularize the target lesion (without any sequelae) to acute mortality. Interestingly, myocardial infarction was used in each definition; however, in some studies, the definition was limited to Q-wave infarction. All-cause mortality was used in 11 of the 20 studies, while cardiovascular death was used in 11 studies; both definitions were used in only two studies. In this particular meta-analysis, the outcome, whether DES was superior to BMS, was dependent on the definition of MACE. Therefore, a composite must be pre-specified and contain clinically meaningful elements, and every component must be individually presented. The definition is paramount, - not so the name.

We are obligated to ensure that our research dollars are utilized efficiently. Every RCT should result in an accurate database where the characteristics and outcomes are universally defined. This process creates a valuable and recyclable resource going forward. The acknowledgment that we cannot conduct an RCT for every clinical question is fuelling the search for alternative and efficient methods to supply our thirst for reproducible evidence. In this endeavour, the data from RCTs can be utilized to conduct non-randomized cohort studies. This option stems from research that showed striking correlations between the results from 46 meta-analyses of RCTs and the results of comparable subject matter conducted using the nonrandomized design. ${ }^{23}$ This particular study acknowledged that - at least for these clinical questions - non-randomized studies tended to show larger treatment effects. The results of the two types of designs differed beyond chance in $15 \%$ 
of the comparisons. The discrepancies between the RCT and non-randomized trials were less common when only prospective studies were considered. Additionally, sample size and disparate timing between publications were also noted to increase the discrepancies. This type of analysis of clinical care has now been independently reproduced no less than 13 times. These studies have been combined in a meta-analysis collating data from 1,583 separate metaanalyses of RCTs covering 228 different medical conditions. $^{24}$ The conclusions were that no statistical differences in results could be found based on the study design. Further, when the findings of the RCTs were compared with the prospective cohort studies alone, the pooled odds ratio was virtually identical. The ability to independently reproduce a finding is the core principle of quality of evidence. ${ }^{25}$

For the foreseeable future, there will be more demand for evidence than we, as a community with a vested interest, can produce. Importantly, this should not be seen as advocating for a reduction in the number of RCTs; indeed, quite the opposite. But when we are able to conduct an RCT, it needs to be of only the highest quality. This issue of the Journal documents the willingness for clinical trialists to adopt a core set of baseline characteristics and clinically meaningful and universally defined outcomes. ${ }^{26}$ Future collaborations will be expected to produce increasingly accurate and homogeneous databases that can also be recycled in numerous ways. Current and evolving research designs and statistical methods will improve our ability to reduce biases. Highquality evidence will be produced with ever expanding methodology that enhances the role of the RCT. We are optimistic about the future and our ability to translate evidence into high-quality clinical care. The evidence supports this view. What's in a name? Agreement on what constitutes an important clinical outcome and how it should be defined is critical to improving clinical care. Whether we call it Montague or Capulet matters little.

\section{Les défis à relever pour produire des données cliniques probantes de grande qualité}

\author{
« C'est ton nom seul qui est mon ennemi » \\ Romeo and Juliet, Act II Scene II
}

En 1996, David Sackett déclarait que «la médecine fondée sur des données probantes (EBM) signifie l'intégration de l'expertise clinique individuelle avec les meilleures preuves cliniques extérieures disponibles tirées d'une recherche systématique ». Les cliniciens, les enseignants, les investigateurs et les administrateurs cherchant à améliorer les soins cliniques ont adopté ce point de vue et, maintenant, l'EBM alimente des activités qui visent à produire des données cliniques de grande qualité.

L'adoption de l'EBM a entraîné un nombre toujours croissant de lignes directrices pour une pratique optimale, ${ }^{1}$ des règles de paiement en fonction des performances et/ou des politiques publiques. ${ }^{2}$ Malheureusement pour la médecine périopératoire, ce processus de recherche, de transfert du savoir et les changements subséquents de données probantes périopératoires n'a pas produit les améliorations cliniques que beaucoup prédisaient. ${ }^{3}$ On peut avancer de nombreuses explications pour ces échecs, mais, à mon avis, il faut principalement blâmer notre méthodologie de recherche. ${ }^{4}$ L'amélioration de la qualité des investigations cliniques nécessite plus qu'un simple processus de randomisation. ${ }^{5}$ Nous sommes mis au défi de mener des études efficaces, rapides et ayant une puissance suffisante, destinées à élucider des hypothèses biologiquement fondées qui répondront à des questions cliniques importantes et clairement définies.

Tandis que l'EBM idéale est très encouragée, nous nous heurtons en pratique à de nombreux obstacles. Le plus grand problème est bien sûr d'ordre économique : la demande dépasse l'offre. Penser que chaque question clinique, et elles sont innombrables, puisse trouver une réponse grâce à une méta-analyse et/ou des analyses systématiques de multiples essais cliniques randomisés (ECR) est faire preuve de naïveté. En fait, on ne peut citer que quelques exemples d'études en médecine périopératoire ayant mené à des recommandations de la plus grande qualité. ${ }^{6,7}$ Nos échecs sont des exemples de recommandations cliniques basées sur de l'information encline à un bon nombre de biais. ${ }^{8}$ Aujourd'hui, le défi consiste à produire des données probantes de qualité.

L'ECR est devenu synonyme de donnée probante non biaisée. Néanmoins, la possibilité de générer et reproduire un ECR est limitée par une deuxième réalité économique : son financement. Le financement est un frein constant qui limite la recherche clinique périopératoire et entrave donc l'EBM. La nécessité d'un personnel multidisciplinaire, d'une infrastructure, de niveaux croissants de bureaucratie, les questions concernant le respect de la vie privée et le temps nécessaire pour terminer les études ajoutent au coût de réalisation de n'importe quelle étude. En même temps, le montant du financement revu par les pairs à la disposition des investigateurs canadiens stagne ou diminue. ${ }^{9}$ Un autre aspect des dépenses de recherche croissantes est la réalisation que pour atteindre des critères d'évaluation cliniquement importants, les essais 
interventionnels nécessitent des échantillons d'étude suffisamment grands pour saisir des différences reproductibles qui aient du sens; cela veut dire qu'il faudra souvent inclure plusieurs milliers de patients dans un essai. ${ }^{10}$ Dans Transforming Clinical Research in the United States: Challenges and Opportunities, les auteurs ont montré que des essais interventionnels internationaux pouvaient coûter plus de 300 millions de dollars. ${ }^{11}$ En vérité, ce chiffre surestime de plus de 20 fois les coûts des essais périopératoires récents; néanmoins les coûts vont continuer à augmenter et les opportunités doivent être optimisées.

Ce numéro spécial du Journal reconnaît qu'une étape importante est faite dans cette direction, avec un débat pour définir des critères d'évaluation cliniquement importants et centrés sur le patient. ${ }^{12,13}$ La méta-analyse d'ERC bien menée est mise au pinacle des niveaux de preuves : en d'autres termes, des constatations qui sont reproductibles. ${ }^{14}$ Les méta-analyses futures seront plus fortes quand chaque essai examinera un ensemble de critères d'évaluation universellement acceptés.

Pourquoi y a-t-il un problème? La majorité des cliniciens supposent que les critères d'évaluation sont noirs ou blancs, c'est-à-dire que nous savons ce qu'ils signifient et lesquels sont importants. Curieusement, les définitions objectives de nombreux critères d'évaluation cliniques sont ambiguës, et cette ambiguïté peut entraîner une mauvaise interprétation. Voyons la définition du décès qui pourrait nous sembler le critère d'évaluation le plus facile à définir. Néanmoins, est-il important de rassembler les décès survenus à l'hôpital ou les décès survenus 30 jours après l'intervention? Dans les essais cardiovasculaires, par exemple, est-il important de faire la distinction entre décès de cause cardiaque et les décès de cause non cardiaque? Si oui, comment définiriez-vous un décès d'origine cardiaque? Le définiriez-vous comme étant l'absence d'autres causes ayant pu survenir sans être identifiées ou est-il nécessaire d'utiliser des données objectives (peut-être un biomarqueur)? Actuellement, ces types de questions restent sans réponse et ne font l'objet d'aucune forme de consensus. Il n'est donc pas surprenant de trouver des résultats discordants lorsque les caractéristiques à l'inclusion et les critères d'évaluation postopératoires de deux projets majeurs d'amélioration de la qualité (c'est-à-dire, le National Surgical Quality Improvement Program et la Vascular Quality Initiative) ont été appliqués à une même base de données. Inutile d'aller plus loin pour illustrer le besoin pressant de définir les critères d'évaluation cliniquement importants les plus acceptés. ${ }^{15}$ Même si nous en savons le nom (c'est-à-dire, le nom du critère d'évaluation), sa définition est de toute évidence plus importante.

De façon universelle, les plus importants critères d'évaluation cliniques centrés sur le patient sont souvent les moins fréquents. Cette réalité peut rendre la réalisation d'une étude impossible simplement en raison de la taille de l'échantillon nécessaire. Les investigateurs doivent donc utiliser des critères de substitution et/ou des critères composites pour réduire la taille de la cohorte étudiée dans la majorité des essais modernes. Bien que ces instruments soient à la fois courants et nécessaires, les investigateurs se sont heurtés à des difficultés quand ils les ont utilisés pour interpréter les résultats de nombreux essais.

Par exemple, les critères d'insuffisance rénale aiguë de l'AKIN (Acute Kidney Injury Network) ou RIFLE (Risk Injury FaiLurE) sont des cas connus de critères d'évaluation de substitution utilisant des modifications objectives des biomarqueurs (créatinine et diurèse) pour définir les critères d'évaluation. ${ }^{16}$ Les critères AKIN et RIFLE sont tous deux acceptés depuis que des études observationnelles prospectives ont montré des associations significatives avec des aboutissements importants quoique rares, tels que la dialyse et le décès. L'ampleur de ces associations est illustrée dans une méta-analyse pour évaluer la variabilité et la précision des critères AKIN. Dans cette étude de huit essais et plus de 50000 patients, une lésion rénale aiguë légère, définie par une augmentation de la créatinine de plus de $25 \mathrm{mmol} \cdot \mathrm{L}^{-1}$ (le critère de substitution) a été constatée chez $17 \%$ des patients. ${ }^{17} \mathrm{Ce}$ degré d'augmentation de la créatinine a été associé à un doublement significatif des décès proches du terme. Cependant, le taux de décès observé dans cette analyse était de 1,2\% (619 patients), une incidence 14 fois plus faible que l'insuffisance rénale selon la définition de l'AKIN. Dans la mesure où les taux d'événements du critère d'évaluation de substitution sont plus fréquents, il est possible de recruter un échantillon de plus petite taille, mais nous ne devons pas oublier que plus de $95 \%$ des patients ayant éprouvé le critère de substitution n'ont pas présenté le critère de base.

Il est également possible de recruter de plus petites cohortes quand un critère composite est utilisé comme critère d'évaluation principal. De plus, l'utilisation de critères composites exige un examen méticuleux et une interprétation prudente des résultats. Le nom (du composite) peut-être le même, mais la façon dont il est défini est souvent inhomogène et ouverte à des manipulations pouvant conduire à des résultats déroutants. ${ }^{18}$ Les critères composites sont souvent construits a posteriori et peuvent inclure des facteurs dont l'importance clinique est très variable, produisant des résultats biaisés ou contradictoires. ${ }^{19,20}$

Un bel exemple d'un tel critère est l'EICM ou « événements indésirables cardiaques majeurs » (MACE - major adverse cardiac events). ${ }^{21}$ l'EICM est populaire et largement utilisé, mais ses composants varient énormément d'une étude à l'autre. Une revue systématique comparant l'efficacité des endoprothèses à libération de produit actif 
(stent à élution de médicament) et des endoprothèses en métal non recouvert ${ }^{22}$ a trouvé dix composants différents utilisés pour définir l'EICM, avec un minimum de trois composants et un maximum de sept. En termes d'importance clinique, les facteurs allaient de la nécessité de revasculariser la lésion cible (sans aucune séquelle) à la mortalité aiguë. Il est intéressant de noter que l'infarctus du myocarde était utilisé dans chaque définition; cependant dans certaines études la définition se limitait à la présence d'une onde Q. La mortalité toutes causes a été utilisée dans 11 des 20 études, tandis que la mortalité cardiovasculaire a été utilisée dans 11 études; les deux définitions n'ont été utilisées que dans deux études. Dans cette méta-analyse particulière cherchant à savoir si les endoprothèses à élution de médicament actif étaient supérieures aux endoprothèses en métal nu, le critère d'évaluation reposait sur la définition de l'EICM. En conséquence, un critère composite doit être préspécifié et contenir des éléments cliniquement pertinents; en outre, chacun de ses composants doit être présenté de manière individuelle. La définition est primordiale, bien plus que le nom.

Nous avons l'obligation de nous assurer que nos dollars consacrés à la recherche sont utilisés de manière efficace. Tous les essais cliniques randomisés doivent se traduire par une base de données exacte où les caractéristiques et critères d'évaluation sont définis de façon universelle. Cette procédure crée une ressource valable et réutilisable pour aller de l'avant. Admettre que nous ne pouvons pas mener d'ECR pour chaque question clinique alimente la recherche de méthodes alternatives et efficaces pour étancher notre soif de données probantes reproductibles. Dans cette tentative, les données provenant des ECR peuvent servir à mener des études de cohortes non randomisées. Cette possibilité ressort de recherches qui ont montré des corrélations frappantes entre les résultats de 46 méta-analyses d'essais cliniques randomisés et les résultats d'études non randomisées sur des sujets comparables. ${ }^{23}$ Cette étude particulière a reconnu que les études non randomisées tendaient à montrer des effets thérapeutiques plus importants, au moins pour ce qui avait trait à ces questions cliniques. Les résultats de ces deux types de schémas présentaient des différences non liées au hasard dans $15 \%$ des comparaisons. Les divergences entre les essais cliniques randomisés et les essais non randomisés étaient moins fréquentes quand les études prospectives étaient les seules prises en compte. De plus, on a également noté que la taille de l'échantillon et les écarts dans le temps entre les publications augmentaient les divergences. Ce type d'analyse des soins a maintenant été reproduit de façon indépendante au moins 13 fois. Ces études ont été combinées dans une méta-analyse collectant les données de 1583 méta-analyses distinctes d'essais cliniques randomisés couvrant 228 maladies différentes. ${ }^{24}$ Ses conclusions ont été que les différences statistiques entre les résultats pouvaient être dues au plan de l'étude. De plus, lorsque les constatations des ECR ont été comparées à celles des études de cohortes prospectives seules, les rapports de cotes groupées ont été pratiquement identiques. La capacité à reproduire de façon indépendante une constatation est le principe central de la qualité des données probantes. ${ }^{25}$

Il y aura, dans un avenir prévisible, plus de demandes de données probantes que nous - communauté professionnelle ayant un intérêt particulier — ne pourrons produire. Il est important de ne pas utiliser cet argument pour plaider en faveur d'une réduction du nombre d'ECR, mais plutôt pour le contraire. Cependant, quand nous sommes en mesure de mener un ECR, cela ne doit être qu'un essai de la plus grande qualité. Ce numéro du Journal documente le désir d'adopter, pour les essais cliniques, un ensemble de base des caractéristiques à l'inclusion et de critères d'évaluation cliniquement pertinents, avec une définition universelle. $^{26}$ De futures collaborations devront produire des bases de données de plus en plus précises et homogènes qui pourront être « recyclées » de nombreuses manières. Les plans actuels et évolutifs de la recherche et des méthodes statistiques amélioreront notre capacité à réduire les biais. Des données probantes de grande qualité seront produites avec une méthodologie en progression constante favorisant le rôle des ECR. Nous sommes optimistes pour l'avenir et pour notre capacité à traduire des données probantes en soins cliniques de grande qualité. Les preuves soutiennent ce point de vue.

Qu'y a-t-il dans un nom? Un accord sur ce qui constitue un critère d'évaluation clinique important et sur la façon dont il doit être défini est essentiel pour l'amélioration des soins. Peu importe que nous l'appelions Montagu ou Capulet.

Conflicts of interest None declared.

Funding Dr. Beattie is supported by the R. Fraser Elliot Chair in Cardiac Anesthesia at the University Health Network as well as a peer-reviewed Merit Award from the University of Toronto, Department of Anesthesia.

Conflits d'intérêts Aucun déclaré.

Financement Le Dr Beattie est soutenu par la chaire Fraser Elliot en anesthésie cardiaque du Réseau universitaire de santé ainsi que par un prix de mérite du département d'anesthésie de l'Université de Toronto.

\section{References}

1. Fleisher LA, Beckman JA, Brown KA, et al. 2009 ACCF/AHA focused update on perioperative beta blockade incorporated into the ACC/AHA 2007 guidelines on perioperative cardiovascular evaluation and care for noncardiac surgery. J Am Coll Cardiol 2009; 54: e13-118. 
2. Ryan AM. Effects of the Premier Hospital Quality Incentive Demonstration on Medicare patient mortality and cost. Health Serv Res 2009; 44: 821-42.

3. Prielipp RC, Coursin DB. All That glitters is not a golden recommendation. Anesth Analg 2015; 121: 727-33.

4. Beattie WS. Great expectations: a case for expanded clinical investigation. Anesth Analg 2015; 121: 586-8.

5. Neuman MD, Bosk CL, Fleisher LA. Learning from mistakes in clinical practice guidelines: the case of perioperative betablockade. BMJ Qual Saf 2014; 23: 957-64.

6. Gan TJ, Diemunsch P, Habib AS, et al. Consensus guidelines for the management of postoperative nausea and vomiting. Anesth Analg 2014; 118: 85-113.

7. Wijeysundera DN, Duncan D, Nkonde-Price C, et al. Perioperative beta blockade in noncardiac surgery: a systematic review for the 2014 ACC/AHA guideline on perioperative cardiovascular evaluation and management of patients undergoing noncardiac surgery: a report of the American College of Cardiology/American Heart Association Task Force on practice guidelines. J Am Coll Cardiol 2014; 64: 2406-25.

8. Ioannidis JP. Why most published research findings are false. PLoS Med 2005; 2: e124.

9. Drinjakovic J. Funding Changes Usher in a Dark Age for Canadian Science. The Globe and Mail-December 2014. Available from URL: http://www.theglobeandmail.com/news/ national/funding-changes-usher-in-a-dark-age-for-canadian-science/ article22100092/ (accessed November 2015).

10. Ioannidis $J P$. Contradicted and initially stronger effects in highly cited clinical research. JAMA 2005; 294: 218-28.

11. English $R$, Lebovitz $Y$, Griffin $R$, et al. Transforming Clinical Research in the United States: Challenges and Opportunities: Workshop Summary (2010). The National Academies Press. Available from URL: www.nap.edu/catalog/12900.html (accessed November 2015).

12. COMET Initiative. Available from URL: http://www.cometinitiative.org (accessed November 2015).

13. Blazeby JM, Williamson PR, Altman D. The need for consensus, consistency, and core outcome sets in perioperative research. Can J Anesth 2016; 63: DOI: 10.1007/s12630-015-0529-2.

14. Ioannidis JP. How to make more published research true. PLoS Med 2014; 11: e1001747.

15. Aiello FA, Shue B, Kini N, et al. Outcomes reported by the Vascular Quality Initiative and the National Surgical Quality
Improvement Program are not comparable. J Vasc Surg 2014; 60: 152-9; 159 e 1-3.

16. Thomas ME, Blaine C, Dawnay A, et al. The definition of acute kidney injury and its use in practice. Kidney Int 2015; 87: 62-73.

17. Coca SG, Peixoto AJ, Garg AX, Krumholz HM, Parikh CR. The prognostic importance of a small acute decrement in kidney function in hospitalized patients: a systematic review and metaanalysis. Am J Kidney Dis 2007; 50: 712-20.

18. Cordoba G, Schwartz L, Woloshin S, Bae H, Gotzsche PC. Definition, reporting, and interpretation of composite outcomes in clinical trials: systematic review. BMJ 2010; 341: c3920.

19. Ferreira-Gonzalez I, Busse JW, Heels-Ansdell D, et al. Problems with use of composite end points in cardiovascular trials: systematic review of randomised controlled trials. BMJ 2007; 334: 786

20. Ogawa H, Nakayama M, Morimoto T, et al. Low-dose aspirin for primary prevention of atherosclerotic events in patients with type 2 diabetes: a randomized controlled trial. JAMA 2008; 300: 2134-41.

21. Royo MB, Fleisher LA. Chasing myocardial outcomes: perioperative myocardial infarction and cardiac troponin. Can J Anesth 2016; 63: DOI: 10.1007/s12630-015-0539-0.

22. Kip KE, Hollabaugh $K$, Marroquin OC, Williams DO. The problem with composite end points in cardiovascular studies: the story of major adverse cardiac events and percutaneous coronary intervention. J Am Coll Cardiol 2008; 51: 701-7.

23. Ioannidis JP, Haidich AB, Pappa $M$, et al. Comparison of evidence of treatment effects in randomized and nonrandomized studies. JAMA 2001; 286: 821-30.

24. Anglemyer A, Horvath HT, Bero L. Healthcare outcomes assessed with observational study designs compared with those assessed in randomized trials. Cochrane Database Syst Rev 2014; 4: MR000034.

25. Neuman MD, Goldstein JN, Cirullo MA, Schwartz JS. Durability of class I American College of Cardiology/American Heart Association clinical practice guideline recommendations. JAMA 2014; 311: 2092-100.

26. Boney O, Moonesinghe SR, Myles PS, Grocott MP. Standardizing endpoints in perioperative research. Can J Anesth 2016; 63: DOI: 10.1007/s12630-015-0538-1. 